\title{
Corrigendum
}

\section{Obesity and healthy aging: social, functional and mental well-being among older Canadians}

This corrigendum is being published to correct a number of errors and imprecisions, on pages 437-442, of the following article:

Rao DP, Patel P, Roberts KC, Thompson W. Obesity and healthy aging: social, functional and mental well-being among older Canadians. Health Promot Chronic Dis Prev Can. 2018;38(12):437-44. doi: 10.24095/hpcdp.38.12.01.

\section{1. p. 437 (Abstract, Results)}

\section{Before correction}

While happiness and life satisfaction were not associated with obesity status, older females living with obesity reported negative impressions of whether their aging was healthy.

\section{After correction}

While happiness and life satisfaction were not associated with obesity status, older females living with obesity reported negative impressions of whether their aging was healthy, to a greater extent than males.

\section{2. p. 439 (Methods, Variables, Mental health and well-being)}

\section{Before correction}

We determined mental health status based on self-report of a mood or anxiety disorder diagnosed by a physician. Various measures were used for mental wellbeing. We identified happiness as feeling happy 3 or more days per week versus fewer. While this measure is not a substitute for specific mental health assessment, it has been validated as a useful one to measure general mental health. ${ }^{28}$ Self-reported measures of happiness have been shown to associate with lower mortality, which may be mediated by physical activity and comorbidity, in the elderly. ${ }^{29}$ Life satisfaction was derived from reports of feeling slightly satisfied or better with life versus neutral or dissatisfied. These measures (self-rated mental health and self-rated healthy aging) were each coded as binary variables based on self-report responses of "fair" or "poor" versus "good," "very good" or "excellent," respectively.

\section{After correction}

We determined mental health status based on self-report of a mood or anxiety disorder diagnosed by a physician. Various measures were used for mental wellbeing. We identified happiness as feeling happy 3 or more days per week versus fewer. Self-reported measures of happiness have been shown to associate with lower mortality, which may be mediated by physical activity and comorbidity, in the elderly. ${ }^{29}$ Life satisfaction was derived from reports of feeling slightly satisfied or better with life versus neutral or dissatisfied. Respondents were asked the following two questions to assess mental health and healthy aging, respectively: "In general, would you say your mental health is excellent, very good, good, fair, or poor?" and "In terms of your own healthy aging, would you say it is excellent, very good, good, fair, or poor?" While self-rated mental health is not a substitute for specific mental health assessment, it has been validated as a useful one to measure general health. ${ }^{28}$ These measures (self-rated mental health and self-rated healthy aging) were each coded as binary variables based on self-report responses of "fair" or "poor" versus "good," "very good" or "excellent," respectively.

\section{3. p. 439 (Results, $2^{\text {nd }}$ paragraph)}

\section{Before correction}

The consumption of 4 or more alcoholic beverages per week differed significantly between the sexes, and also decreased significantly with increasing age groups. Obesity was significantly higher among males and decreased with age, until age 75 to 85 , where more females lived with obesity than males, despite their own age-related decreases. Finally, significantly more females than males reported having multimorbidity at ages 55 to 64, with differences disappearing by ages 75 to 85 years (Table 1). 


\section{After correction}

The consumption of 4 or more alcoholic beverages per week differed significantly between the sexes, and also across age groups. Statistically significant differences across age groups were found for obesity for both males and females. Obesity was significantly higher among males than females in the 55-64-year age group, while in the 75-85-year age group, the prevalence of obesity was higher among females than males, though this latter result was not statistically significant. Finally, significantly more females than males reported having multimorbidity at ages 55 to 64, with differences disappearing by ages 75 to 85 years (Table 1).

\section{4. p. 439 (Results, $3^{\text {rd }}$ paragraph)}

\section{Before correction}

Reduced physical functioning was strongly associated with obesity for both males and females, with differences between the sexes being significant only among those aged 65 to 74 years old. The strength of this association between reduced physical functioning and obesity increased with age for both sexes. Similarly, impairments in activities of daily life were significantly associated with obesity for both sexes, with the strength of association increasing with age. The difference between sexes was significant across all age group, with females living with obesity reporting more impairments than males living with obesity.

\section{After correction}

Reduced physical functioning was strongly associated with obesity for both males and females, with differences between the sexes being significant only among those aged 65 to 74 years old. Similarly, impairments in activities of daily life were significantly associated with obesity for both sexes. The difference between sexes was significant across all age group, with females living with obesity reporting more impairments than males living with obesity.

\section{5.p. 439 (Discussion, $1^{\text {st }}$ paragraph)}

\section{Before correction}

While studies have suggested that poor financial health is linked with disease, ${ }^{32}$ we observed that CLSA participants had strong subjective financial well-being.

\section{After correction}

We observed that among CLSA participants in every age group, a lower proportion of females than males had personal incomes equal to or greater than $\$ 50000$, or lived in their own home. Yet, poor financial health has been linked with disease. ${ }^{32}$ We also observed a higher prevalence of multimorbidity among females, compared to males, in all but the oldest age group.

\section{6. p. 442 (Discussion, $4^{\text {th }}$ paragraph)}

\section{Before correction}

The low perception of good mental health among older females is noteworthy, although this improves with age.

\section{After correction}

The low perception of good mental health among older obese females is noteworthy. 